\title{
Sensitivity, Block Sensitivity, and Certificate Complexity of Unate Functions and Read-Once Functions
}

\author{
Hiroki Morizumi \\ Interdisciplinary Graduate School of Science and Engineering, Shimane University, \\ Shimane 690-8504, Japan \\ morizumi@cis.shimane-u.ac.jp
}

\begin{abstract}
Sensitivity, block sensitivity, and certificate complexity are complexity measures for Boolean functions. In this paper, we prove that these three complexity measures are equal to each other if a Boolean function is a unate function or a read-once function. We also prove $\sqrt{n}$ tight lower bounds for the three complexity measures of read-once functions. As an application of our results, the decision tree complexity of unate functions and read-once functions is upper bounded by the square of the sensitivity of the function.
\end{abstract}

\section{Introduction}

Sensitivity, block sensitivity, and certificate complexity of a Boolean function $f$, denoted by $s(f), b s(f)$ and $C(f)$, respectively, are complexity measures for Boolean functions, and related to other complexity measures including the time complexity of CREW PRAMs and decision tree complexity. A long-standing open problem for these measures is whether or not block sensitivity can be polynomially upper bounded by sensitivity:

$$
b s(f) \leq \operatorname{poly}(s(f)) ?
$$

Although many efforts have been devoted to the open problem as we see later, it is still open. On the other hand, if a function $f$ is a monotone function, it is known that $s(f)=b s(f)=C(f)$ [8]. Our main motivation of this paper is to seek other Boolean function classes such that $s(f)=b s(f)=C(f)$.

In this paper, we prove that $s(f)=b s(f)=C(f)$ for unate functions, which are generalized functions of monotone functions, and for read-once functions over the Boolean operators $\wedge, \vee$ and $\oplus$. We also prove that $\sqrt{n} \leq s(f)(=b s(f)=$ $C(f)$ ) for read-once functions which have $n$ input variables, and the lower bound is tight.

\section{Related Works}

Rubinstein [9] exhibited a Boolean function $f$ which has $b s(f)=\frac{1}{2} s(f)^{2}$. The result has been improved [10]2], although the best known gap is still quadratic. Kenyon and Kutin [7] have proved that $b s(f) \leq \frac{e}{\sqrt{2 \pi}} e^{s(f)} \sqrt{s(f)}$. The upper 
bound has been improved to $b s(f) \leq 2^{s(f)-1} s(f)$ by Ambainis et al. 11. Survey papers 45] include more background for this topic. On the average version of the sensitivity, Impagliazzo and Kabanets [6] have given the tight bound on the average sensitivity of read-once de Morgan formulas.

\section{Preliminaries}

\subsection{Sensitivity, Block Sensitivity, and Certificate Complexity}

Let $f:\{0,1\}^{n} \rightarrow\{0,1\}$ be a Boolean function. For an input $x=\left(x_{1}, x_{2}, \ldots, x_{n}\right)$ of $f$ and $S \subseteq[n]=\{1,2, \ldots, n\}$, let $x^{S}$ denotes the input obtained from $x$ by flipping all the bits $x_{i}$ such that $i \in S$. We abbreviate $x^{\{i\}}$ to $x^{i}$. Sensitivity, block sensitivity, and certificate complexity are defined as follows, respectively.

Definition 1. The sensitivity of $f$ on $x$, denoted by $s(f, x)$, is the number of indices $i$ such that $f(x) \neq f\left(x^{i}\right)$. The sensitivity of $f$, denoted by $s(f)$, is $\max _{x} s(f, x)$. For $z \in\{0,1\}$, the $z$-sensitivity of $f$, denoted by $s_{z}(f)$, is $\max _{x \in f^{-1}(z)} s(f, x)$.

Definition 2. The block sensitivity of $f$ on $x$, denoted by bs $(f, x)$, is the maximum number of disjoint subsets $B_{1}, B_{2}, \ldots, B_{b}$ of $[n]$ such that $f(x) \neq f\left(x^{B_{i}}\right)$ for all $i$. The block sensitivity of $f$, denoted by $b s(f)$, is $\max _{x} b s(f, x)$. For $z \in\{0,1\}$, the $z$-block sensitivity of $f$, denoted by $b s_{z}(f)$, is $\max _{x \in f^{-1}(z)} b s(f, x)$.

Definition 3. A certificate of $f$ on $x$ is a subset $S \subseteq[n]$ such that $f(y)=f(x)$ whenever $y_{i}=x_{i}$ for all $i \in S$. The size of a certificate is $|S|$.

The certificate complexity of $f$ on $x$, denoted by $C(f, x)$, is the size of a smallest certificate of $f$ on $x$. The certificate complexity of $f$, denoted by $C(f)$, is $\max _{x} C(f, x)$. For $z \in\{0,1\}$, the $z$-certificate complexity of $f$, denoted by $C_{z}(f)$, is $\max _{x \in f^{-1}(z)} C(f, x)$.

We can easily show the following relation between $s(f), b s(f)$ and $C(f)$.

Proposition 1. For any Boolean function $f$,

$$
s(f) \leq b s(f) \leq C(f) .
$$

Proof. By the definitions of $s(f)$ and $b s(f), s(f) \leq b s(f)$. For all $x$, since a certificate on $x$ have to contain indices of at least one variable of each sensitive block, $b s(f, x) \leq C(f, x)$. Thus, $b s(f) \leq C(f)$.

Let $x_{i}, y_{i} \in\{0,1\}$ for $1 \leq i \leq n$. A Boolean function is called monotone if $f\left(x_{1}, x_{2}, \ldots, x_{n}\right) \leq f\left(y_{1}, y_{2}, \ldots, y_{n}\right)$ whenever $x_{i} \leq y_{i}$ for all $1 \leq i \leq n$. Nisan [8] showed the following proposition for monotone functions.

Proposition 2 ([8]). If $f$ is a monotone function, then

$$
s(f)=b s(f)=C(f) .
$$




\subsection{Unate Functions and Read-Once Functions}

A Boolean function $f:\{0,1\}^{n} \rightarrow\{0,1\}$ is positive unate in $x_{i}, 1 \leq i \leq n$, if

$$
\begin{aligned}
& f\left(x_{1}, \ldots, x_{i-1}, 0, x_{i+1}, \ldots, x_{n}\right) \\
\leq & f\left(x_{1}, \ldots, x_{i-1}, 1, x_{i+1}, \ldots, x_{n}\right)
\end{aligned}
$$

for all $x_{j}, j \neq i$, and is negative unate in $x_{i}$ if

$$
\begin{aligned}
& f\left(x_{1}, \ldots, x_{i-1}, 0, x_{i+1}, \ldots, x_{n}\right) \\
\geq & f\left(x_{1}, \ldots, x_{i-1}, 1, x_{i+1}, \ldots, x_{n}\right)
\end{aligned}
$$

for all $x_{j}, j \neq i$. A function $f$ is called unate if $f$ is positive or negative unate in all $x_{i}$ for $1 \leq i \leq n$. Monotone functions are a special case of unate functions such that a function is positive unate in all input variables.

A Boolean formula is a rooted binary tree in which each internal node is labeled by the Boolean operators $\wedge, \vee$, or $\oplus$ and each leaf is labeled by a Boolean variable or its negation. A Boolean formula computes a Boolean function in a natural way. A Boolean formula is called read-once if every variable appears exactly once. A read-once Boolean function is a Boolean function that can be represented by a read-once Boolean formula. Notice that we define read-once Boolean functions based on Boolean formulas which have the Boolean operator $\oplus$.

\section{Unate Functions}

In this section, we prove the following theorem.

Theorem 1. If $f$ is a unate function, then

$$
s(f)=b s(f)=C(f) .
$$

$s(f), b s(f)$ and $C(f)$ of a Boolean function $f$ are not changed even if some input variables of $f$ are flipped. More precisely, the following lemma holds.

Lemma 1. Let $f(x)$ be a Boolean function, and let $S \subseteq[n]$. For any $S$, if $g(y)$ is defined as $f\left(y^{S}\right)$, then,

$$
s(f)=s(g), b s(f)=b s(g), C(f)=C(g) .
$$

Proof. It is obvious by the definitions of $s(f), b s(f)$ and $C(f)$.

Proof (of Theorem [1). Let $S=\left\{i \mid f\right.$ is negative unate in $\left.x_{i}, 1 \leq i \leq n\right\}$. We define $g(y)$ as $f\left(y^{S}\right)$, then $g(y)$ is monotone. By Lemma 1,

$$
s(f)=s(g), b s(f)=b s(g), C(f)=C(g) .
$$

By Proposition 2,

$$
s(g)=b s(g)=C(g) .
$$

Hence,

$$
s(f)=b s(f)=C(f) .
$$




\section{Read-Once Functions}

In this section, we prove that $s(f)=b s(f)=C(f)$ for any read-once Boolean function (Theorem 2), and prove that $\sqrt{n} \leq s(f)$ (Corollary 1) and the $\sqrt{n}$ lower bound is tight.

\subsection{Lemma}

Consider a read-once Boolean formula $F$ representing a read-once Boolean function. In $F$, two subformulas which are connected to a same node have no common input variables, since every variable appears exactly once in a read-once Boolean formula. This fact enables us to analyze the sensitivity and certificate complexity of functions computed at each node in $F$.

Lemma 2. Let $f_{1}$ and $f_{2}$ be Boolean functions such that $f_{1}$ and $f_{2}$ have no common input variables, and $f_{1}$ and $f_{2}$ are not constant functions.

If $f=f_{1} \wedge f_{2}$, then

$$
\begin{aligned}
s_{0}(f) & =\max \left\{s_{0}\left(f_{1}\right), s_{0}\left(f_{2}\right)\right\}, \\
C_{0}(f) & =\max \left\{C_{0}\left(f_{1}\right), C_{0}\left(f_{2}\right)\right\}, \\
s_{1}(f) & =s_{1}\left(f_{1}\right)+s_{1}\left(f_{2}\right), \\
C_{1}(f) & =C_{1}\left(f_{1}\right)+C_{1}\left(f_{2}\right) .
\end{aligned}
$$

If $f=f_{1} \vee f_{2}$, then

$$
\begin{aligned}
s_{0}(f) & =s_{0}\left(f_{1}\right)+s_{0}\left(f_{2}\right), \\
C_{0}(f) & =C_{0}\left(f_{1}\right)+C_{0}\left(f_{2}\right), \\
s_{1}(f) & =\max \left\{s_{1}\left(f_{1}\right), s_{1}\left(f_{2}\right)\right\}, \\
C_{1}(f) & =\max \left\{C_{1}\left(f_{1}\right), C_{1}\left(f_{2}\right)\right\} .
\end{aligned}
$$

If $f=f_{1} \oplus f_{2}$, then

$$
\begin{aligned}
s_{0}(f) & =\max \left\{s_{0}\left(f_{1}\right)+s_{0}\left(f_{2}\right), s_{1}\left(f_{1}\right)+s_{1}\left(f_{2}\right)\right\}, \\
C_{0}(f) & =\max \left\{C_{0}\left(f_{1}\right)+C_{0}\left(f_{2}\right), C_{1}\left(f_{1}\right)+C_{1}\left(f_{2}\right)\right\}, \\
s_{1}(f) & =\max \left\{s_{0}\left(f_{1}\right)+s_{1}\left(f_{2}\right), s_{1}\left(f_{1}\right)+s_{0}\left(f_{2}\right)\right\}, \\
C_{1}(f) & =\max \left\{C_{0}\left(f_{1}\right)+C_{1}\left(f_{2}\right), C_{1}\left(f_{1}\right)+C_{0}\left(f_{2}\right)\right\} .
\end{aligned}
$$

Proof. Assume that $f=f_{1} \wedge f_{2}$. We consider that $s_{0}(f)=\max \left\{s_{0}\left(f_{1}\right), s_{0}\left(f_{2}\right)\right\}$. If $s_{0}\left(f_{1}\right) \geq s_{0}\left(f_{2}\right)$, we can assign input variables of $f_{2}$ so that $f_{2}=1$, and independently we can assign input variables of $f_{1}$. Thus, we can confirm that $s_{0}(f)=\max \left\{s_{0}\left(f_{1}\right), s_{0}\left(f_{2}\right)\right\}$.

Similarly, we can confirm all equations by the definitions of sensitivity and certificate complexity. 


\subsection{Equality}

Lemma 2 immediately gives the following lemma.

Lemma 3. Let $f_{1}$ and $f_{2}$ be Boolean functions such that $f_{1}$ and $f_{2}$ have no common input variables, and $f_{1}$ and $f_{2}$ are not constant functions. If

$$
f=f_{1} \wedge f_{2}, f=f_{1} \vee f_{2}, \text { or } f=f_{1} \oplus f_{2},
$$

and

$$
\begin{aligned}
& s_{0}\left(f_{1}\right)=C_{0}\left(f_{1}\right), s_{1}\left(f_{1}\right)=C_{1}\left(f_{1}\right), \\
& s_{0}\left(f_{2}\right)=C_{0}\left(f_{2}\right), s_{1}\left(f_{2}\right)=C_{1}\left(f_{2}\right),
\end{aligned}
$$

then

$$
s_{0}(f)=C_{0}(f), s_{1}(f)=C_{1}(f) .
$$

Now, we prove the following theorem.

Theorem 2. If $f$ is a read-once Boolean function, then

$$
s(f)=b s(f)=C(f) .
$$

Proof. Since $s(f) \leq b s(f) \leq C(f)$ for any Boolean function $f$ by Proposition 1 we only need to prove $s(f)=C(f)$.

Let $n$ be the number of input variables of $f$. We use induction on $n$ and prove $s_{0}(f)=C_{0}(f)$ and $s_{1}(f)=C_{1}(f)$.

Base: $n=1$. Then, $f=x_{1}$ or $f=\neg x_{1}$, and $s_{0}(f)=s_{1}(f)=1$ and $C_{0}(f)=$ $C_{1}(f)=1$. Thus, $s_{0}(f)=C_{0}(f)$ and $s_{1}(f)=C_{1}(f)$.

Induction Step: Suppose $s_{0}\left(f^{\prime}\right)=C_{0}\left(f^{\prime}\right)$ and $s_{1}\left(f^{\prime}\right)=C_{1}\left(f^{\prime}\right)$ for every Boolean function $f^{\prime}$ such that the number of input variables of $f^{\prime}$ is less than $n$.

Let $F$ be a read-once Boolean formula which computes $f$. Recall that we define Boolean formulas as rooted binary trees. Let $f_{1}$ and $f_{2}$ are Boolean functions computed by subformulas which are connected to the root node of $F$. Then, $f=f_{1} \wedge f_{2}, f=f_{1} \vee f_{2}$, or $f=f_{1} \oplus f_{2}$, and the number of input variables of $f_{1}$ and $f_{2}$ is less than $n$, respectively. By the supposition, $s_{0}\left(f_{1}\right)=C_{0}\left(f_{1}\right)$, $s_{1}\left(f_{1}\right)=C_{1}\left(f_{1}\right), s_{0}\left(f_{2}\right)=C_{0}\left(f_{2}\right)$ and $s_{1}\left(f_{2}\right)=C_{1}\left(f_{2}\right)$. Thus, by Lemma 3 $s_{0}(f)=C_{0}(f)$ and $s_{1}(f)=C_{1}(f)$, which mean $s(f)=C(f)$.

\subsection{Lower Bound}

Lemma 2 also gives a lower bound for the sensitivity of read-once functions.

Theorem 3. If $f$ is a read-once Boolean function of $n$ input variables, then

$$
n \leq s_{0}(f) s_{1}(f) .
$$


Proof. We use induction on $n$.

Base: $n=1$. Then, $f=x_{1}$ or $f=\neg x_{1}$, and $s_{0}(f) s_{1}(f)=1$. Thus, $n \leq$ $s_{0}(f) s_{1}(f)$.

Induction Step: Suppose $n^{\prime} \leq s_{0}\left(f^{\prime}\right) s_{1}\left(f^{\prime}\right)$ for every Boolean function $f^{\prime}$ such that the number of input variables of $f^{\prime}$, denoted by $n^{\prime}$, is less than $n$.

Let $F$ be a read-once Boolean formula which computes $f$. Recall that we define Boolean formulas as rooted binary trees. Let $f_{1}$ and $f_{2}$ are Boolean functions computed by subformulas which are connected to the root node of $F$, and let $n_{1}$ and $n_{2}$ are the number of input variables of $f_{1}$ and $f_{2}$, respectively. Then, $f=f_{1} \wedge f_{2}, f=f_{1} \vee f_{2}$, or $f=f_{1} \oplus f_{2}$, and $n_{1}<n, n_{2}<n$, and $n_{1}+n_{2}=n$. By the supposition, $n_{1} \leq s_{0}\left(f_{1}\right) s_{1}\left(f_{1}\right)$ and $n_{2} \leq s_{0}\left(f_{2}\right) s_{1}\left(f_{2}\right)$.

If $f=f_{1} \wedge f_{2}$, then, by Lemma 2 .

$$
\begin{aligned}
s_{0}(f) s_{1}(f) & =\max \left\{s_{0}\left(f_{1}\right), s_{0}\left(f_{2}\right)\right\} s_{1}\left(f_{1}\right)+\max \left\{s_{0}\left(f_{1}\right), s_{0}\left(f_{2}\right)\right\} s_{1}\left(f_{2}\right) \\
& \geq s_{0}\left(f_{1}\right) s_{1}\left(f_{1}\right)+s_{0}\left(f_{2}\right) s_{1}\left(f_{2}\right) \\
& \geq n_{1}+n_{2}=n .
\end{aligned}
$$

Similarly, we can prove that $n \leq s_{0}(f) s_{1}(f)$ also for the cases that $f=f_{1} \vee f_{2}$ and $f=f_{1} \oplus f_{2}$.

Recall that $s(f)=\max \left\{s_{0}(f), s_{1}(f)\right\}$.

Corollary 1. If $f$ is a read-once Boolean function of $n$ input variables, then

$$
\sqrt{n} \leq s(f)
$$

The lower bounds in Theorem 3 and Corollary 1 are tight, since we can easily confirm that the following read-once Boolean function $f$ has $s_{0}(f)=n / m$ and $s_{1}(f)=m$. (We assume that $m$ is a positive integer such that $n / m$ becomes an integer.)

$$
f=\bigvee_{i=1}^{n / m} \bigwedge_{j=1}^{m} x_{m(i-1)+j}
$$

\section{Concluding Remarks}

In this paper, we investigated the sensitivity, block sensitivity, and certificate complexity of unate functions and read-once functions. As the conclusion of this paper, we show an application of our results to decision tree complexity.

Let $D(f)$ denote the decision tree complexity of $f$, i.e., the depth of an optimal decision tree that computes $f$. Beals et al. [3] prove

Theorem 4 ([3]). For any Boolean function $f$,

$$
D(f) \leq C_{1}(f) b s(f) .
$$


Recall that we proved that $s(f)=b s(f)=C(f)$ for any unate function $f$ (Theorem 10) and for any read-once function $f$ (Theorem 2), and $C_{1}(f) \leq C(f)$ by the definition. Thus, we obtain the following corollary.

Corollary 2. If $f$ is a unate function or a read-once function, then

$$
D(f) \leq s(f)^{2}
$$

Although Corollary 2 is meaningful for unate functions, we have to be attentive for read-once functions, since we can easily see that $D(f)=n$ for every read-once function. Thus, Corollary 2 is an alternating proof of Corollary 1 rather than an upper bound of $D(f)$. Notice that the alternating proof depends on Theorem 4 and cannot prove Theorem 3.

\section{References}

1. Ambainis, A., Gao, Y., Mao, J., Sun, X., Zuo, S.: New upper bound on block sensitivity and certificate complexity in terms of sensitivity. CoRR abs/1306.4466 (2013)

2. Ambainis, A., Sun, X.: New separation between $s(f)$ and $b s(f)$. Electronic Colloquium on Computational Complexity (ECCC) 18, 116 (2011)

3. Beals, R., Buhrman, H., Cleve, R., Mosca, M., de Wolf, R.: Quantum lower bounds by polynomials. J. ACM 48(4), 778-797 (2001)

4. Buhrman, H., de Wolf, R.: Complexity measures and decision tree complexity: a survey. Theor. Comput. Sci. 288(1), 21-43 (2002)

5. Hatami, P., Kulkarni, R., Pankratov, D.: Variations on the sensitivity conjecture. Theory of Computing, Graduate Surveys 2, 1-27 (2011)

6. Impagliazzo, R., Kabanets, V.: Fourier concentration from shrinkage. Electronic Colloquium on Computational Complexity (ECCC) 20, 163 (2013)

7. Kenyon, C., Kutin, S.: Sensitivity, block sensitivity, and l-block sensitivity of boolean functions. Inf. Comput. 189(1), 43-53 (2004)

8. Nisan, N.: CREW PRAMs and decision trees. SIAM J. Comput. 20(6), 999-1007 (1991)

9. Rubinstein, D.: Sensitivity vs. block sensitivity of boolean functions. Combinatorica 15(2), 297-299 (1995)

10. Virza, M.: Sensitivity versus block sensitivity of boolean functions. Inf. Process. Lett. 111(9), 433-435 (2011) 